Pacific Journal of Mathematics

WNEREALIX NELLCAPPED CONE 


\section{UNIVERSALLY WELL-CAPPED CONES}

\section{Asimow}

A closed convex cone $P$ is said to be universally wellcapped if it contains a compact convex subset $B$ such that $P \backslash B$ is convex and $P=\cup_{n=1}^{\infty} n B$. The compact convex sets which are universal caps of some cone are represented as the positive part of the unit ball of an ordered Banach dual space with the weak* topology. A characterization, involving the directedness of the unit ball, is given of those ordered Banach spaces whose dual cones are universally well-capped. An application is made to the Choquet boundary theory for subspaces of continuous functions on a compact Hausdorff space.

The notion of a cap of a convex set $X$ was introduced by Choquet [2] for the case where $X$ is a cone in a Hausdorff locally convex space $E$. Following Choquet, $B$ is a $\operatorname{cap}$ of $X$ if $B$ is a compact convex subset of $X$ for which $X \backslash B$ is convex. If each point of $X$ is contained in a cap then $X$ is said to be well-capped. An important property of well-capped closed convex sets is that they satisfy a Krein-Milman type theorem $[1 ; 2]$.

If $B$ is a cap of a cone $Q$ such that $Q=\bigcup_{n=1}^{\infty} n B$ then $B$ is called a universal cap of $Q$. It is shown in [1] that if $X$ is closed, convex and well-capped then $X \times\{1\}$ generates a closed convex wellcapped cone $Q$ in the space $E \times R$. Each cap of $X$ is associated with a cap of the cone $Q$ and each cap $B$ of $Q$ is itself a universal cap of the extremal sub-cone $\bigcup_{n=1}^{\infty} n B$. The purpose of this paper is to give a characterization of those compact convex sets which are universal caps of some cone. As far as we know, this problem was first posed by Choquet in lectures given at the University of Washington in 1964.

A particular instance of a universally capped cone is the case where the cone has a compact base. It is shown by Klee [11] that this is equivalent to the cone being locally compact. The properties of locally compact cones have been studied in some detail. We refer specifically to the work of D.A. Edwards [5] in which he shows that a locally compact cone can be embedded in a Banach space with the weak* topology as the dual of a Banach space with an order-unit norm.

We note here that an analogous construction is possible for universally capped cones. We give a characterization of the sub-dual spaces that arise in this context in terms of an ordering property of the unit ball which we term approximate directedness (definition 
below). It is shown that a set is a universal cap of a cone if and only if it is linearly homeomorphic to the positive part of the unit ball (weak* topology) in the dual of an approximately directed Banach space.

If $E$ is an approximately directed subspace of continuous functions on a compact Hausdorff space then $E$ possesses a Šilov boundary. Since such subspaces need not contain the constants this allows an extension of the standard minimal boundary theory to a class of continuous functions vanishing at infinity on a locally compact Hausdorff space.

2. Approximately directed Banach spaces. Let $E$ be a Banach space ordered by the closed convex positive cone $P$. In what follows we shall denote the dual of $E$ by $E^{*}$ and the set $\{x \in E:\|x\| \leqq a\}$ by $E_{\alpha}$.

Definition. The ordered Banach space $E$ is said to be $\alpha$-directed if whenever $x$ and $y$ are elements of $E_{1}$, there exists $z \in E_{\alpha}$ such that $z \geqq x$ and $z \geqq y$. If $E$ is 1 -directed we will say simply that $E$ is directed. If $E$ is $\alpha$-directed for all $\alpha>1$ we will say that $E$ is approximately directed. We give an example below (§5) of an approximately directed space which is not directed.

Definition. Let $E$ be a Banach space with a closed positive cone $P$. Define the dual cone

$$
P^{*} \equiv\left\{F \in E^{*} \mid F(x) \geqq 0 \text { for all } x \in P\right\} .
$$

Denote by $B$ the positive part of the unit ball in $E^{*}$, i.e., $B=$ $P^{*} \cap E_{1}^{*}$. The set $B$ is always compact in the weak* topology.

THEOREM 1. The following are equivalent:

(i) $E$ is approximately directed

(ii) $B$ is a universal cap of $P^{*}$ in the weak* topology

(iii) The norm in $E^{*}$ is additive on $P^{*}$.

Proof. ( i ) $\Rightarrow$ (ii). Clearly $B$ is compact, convex and $P^{*}=$ $\bigcup_{n=1}^{\infty} n B$. Suppose $F$ and $G$ are elements of $P^{*} \backslash B$. Then $\|F\|$, $\|G\|>1$. Choose $\alpha>1$ and $f, g \in E_{1}$ such that $F(f)>\alpha$ and $G(g)>\alpha$. Since $E$ is $\alpha$-directed there exists $h \in E_{\alpha}$ such that $h \geqq f, g$. If $0 \leqq \lambda \leqq 1$ then

$$
\begin{aligned}
\|\lambda F+(1-\lambda) G\| \alpha & \geqq\|\lambda F+(1-\lambda) G\| \cdot\|h\| \\
& \geqq \lambda F(h)+(1-\lambda) G(h)>\alpha .
\end{aligned}
$$


Thus $\|\lambda F+(1-\lambda) G\|>1$ and so $P^{*} \backslash B$ is convex.

(ii) $\Rightarrow$ (iii). This follows immediately from the convexity of $P^{*} \backslash B$.

To show (iii) $\Rightarrow$ (i) we first state a lemma.

Lemma. Assume the norm in $E^{*}$ is additive on $P^{*}$. Let $h$ be an affine function on $E^{*}$ such that $h(0)>0$. Assume further that $h \mid E_{1}^{*}$ is weak* continuous. Define $\bar{h}$ on $E^{*}$ by $\bar{h}(0)=0$ and

$$
\bar{h}(x)=\|x\| \cdot h(x /\|x\|) \text { for } x \neq 0 \text {. }
$$

Then $\bar{h} \mid E_{1}^{*}$ is convex, lower-semi-continuous, and $\bar{h} \mid B$ is affine. Also $\bar{h}\left|E_{1}^{*} \leqq h\right| E_{1}^{*}$.

Proof of lemma. Observe first that if $x \neq 0$

$$
h(x)=\|x\| \cdot h(x /\|x\|)+(1-\|x\|) \cdot h(0) .
$$

Thus for all $x, h(x)=\bar{h}(x)+(1-\|x\|) h(0)$ and it follows that $h(x)-h(0) \leqq \bar{h}(x)$ with equality if and only if $x=0$ and

$$
\begin{aligned}
& \bar{h}(x)<h(x) \text { if }\|x\|<1 \\
& \bar{h}(x)=h(x) \text { if }\|x\|=1 \\
& \bar{h}(x)>h(x) \text { if }\|x\|>1 .
\end{aligned}
$$

We show first $\bar{h}$ is convex on $E_{1}^{*}$. Let $x, y \in E_{1}^{*}$ and $z=\lambda x+(1-\lambda) y$; $0<\lambda<1$. Suppose first $x, y, z \neq 0$. Let $a=\lambda\|x\|+(1-\lambda)\|y\|$. Then $a \geqq\|z\|$ with equality if $x, y \in B$. Then

$$
\begin{aligned}
\bar{h}(z) & =\|z\| h(z /\|z\|) \leqq\|z\| h(z /\|z\|)+(a-\|z\|) h(0) \\
& =a[(\|z\| / a) h(z /\|z\|)+((a-\|z\|) \| / a) h(0)] \\
& =a h(z / a) \\
& =a h[(\lambda\|x\| / a)(x /\|x\|)+((1-\lambda)\|y\| / a)(y /\|y\|)] \\
& =\lambda \bar{h}(x)+(1-\lambda) \bar{h}(y)
\end{aligned}
$$

with equality if $x, y \in B$. The cases where $x, y$ or $z=0$ follow similarly. Thus $\bar{h}$ is convex on $E_{1}^{*}$ and affine on $B$.

To show $\bar{h}$ is weak* l.s.c. on $E_{1}^{*}$ it suffices to show

$$
A \equiv\left\{(x, r) \in E_{1}^{*} \times R: \bar{h}(x) \leqq r\right\}
$$

is closed. Let

$$
C=\left\{(x, r) \in E_{1}^{*} \times R: h(x)=r\right\}
$$

and

$$
C^{\prime}=\left\{(x, r) \in E_{1}^{*} \times R: h(x) \leqq r\right\}
$$


Then $C$ is compact, $C^{\prime}$ is closed and we show that

$$
A=C^{\prime} \cup \operatorname{conv}(C \cup\{0,0\}) \text {. }
$$

Let $(x, r) \in A$. If $h(x) \leqq r$ then $(x, r) \in C$. Suppose then $\bar{h}(x) \leqq r<h(x)$ and $x \neq 0$. Then $\bar{h}(x)>h(x)-h(0)$, so $h(x)>r>(h(x)-h(0))$. Let $s=h(0) /(h(0)-h(x)+r)$. Then $1<s<\infty$. Since

$$
x=(1 / s)(s x)+((s-1) / s) 0, h(x)=(1 / s) h(s x)+((s-1) / s) h(0) .
$$

It follows from the last equation and the definition of $s$ that $h(s x)=s r$. Also $\bar{h}(s x)=s\|x\| h(x /\|x\|)=s \bar{h}(x) \leqq s r=h(s x)$ and thus $\|s x\| \leqq 1$. So we have

$$
(x, r)=(1 / s)(s x, s r)+((s-1) / s)(0,0) \in \operatorname{conv}(C \cup\{0,0\}) .
$$

If $(x, r) \in C^{\prime}$ then $(x, r) \in A$. If $(x, r)=\lambda(y, s)$ where $(y, s) \in C$ and $0<\lambda<1$, we have $\bar{h}(x)=\lambda \bar{h}(y) \leqq \lambda h(y)=\lambda s=r$. Thus $(x, r) \in A$.

We continue now with the proof of (iii) $\Rightarrow$ (i). Let $\alpha>1$ and $f, g \in E_{1}$ be given and let $\varepsilon=\alpha-1$. We show that there exists a sequence $\left\{h_{n}\right\}_{n=1}^{\infty}$ of weak* continuous affine functions on $E^{*}$ such that

$$
\begin{gathered}
h_{n}(x) \geqq f(x) \vee g(x) \text { for all } x \in B, \\
0<h_{n}(0)<\varepsilon 2^{-n}, \\
\sup \left\{\left|h_{n}(x)\right|: x \in E_{1}^{*}\right\} \leqq 1+\varepsilon \sum_{k=1}^{n} 2^{-k} \\
\sup \left\{\left|h_{n}(x)-h_{n-1}(x)\right|: x \in E_{1}\right\} \leqq 5 \varepsilon 2^{-n} \text { for } n>1 .
\end{gathered}
$$

Let

$$
\begin{aligned}
H_{0} & =\left\{(x, r) \in E_{1}^{*} \times R: r \geqq\|x\|+\varepsilon / 2\right\}, \\
B_{1} & =\{(x, r) \in B \times R: f(x)=r\}, \\
B_{2} & =\{(x, r) \in B \times R: g(x)=r\}
\end{aligned}
$$

and let $A \equiv \operatorname{conv}\left(B_{1} \cup B_{2}\right)$. With the weak* topology on $E^{*}, H_{0}$ is closed and convex, and $A$ is compact and convex. Also if $(x, r) \in A$,

$$
\begin{array}{r}
(x, r)=\lambda\left(x_{1}, r_{1}\right)+(1-\lambda)\left(x_{2}, r_{2}\right) ; 0 \leqq \lambda \leqq 1, f\left(x_{1}\right)=r_{1}, \\
g\left(x_{2}\right)=r_{2} .
\end{array}
$$

Since $\|\cdot\|$ is affine on $B$,

$$
\|x\|=\lambda\left\|x_{1}\right\|+(1-\lambda)\left\|x_{2}\right\| \geqq \lambda f\left(x_{1}\right)+(1-\lambda) g\left(x_{2}\right)=r
$$

and so $H_{0}$ and $A$ are disjoint. Using the separation theorem on $E^{*} \times R$ and taking the hyperplane so obtained as the graph of a function on $E^{*}$ we have a weak* continuous affine function $h_{1}$ such that

$$
h_{1}(x) \geqq f(x) \vee g(x) \text { for } x \in B \text {, and } 0<h_{1}(0)<\varepsilon / 2 \text {. }
$$


Since

$$
h_{1}(x) \leqq\|x\|+\varepsilon / 2, h_{1}(x)=2 h_{1}(0)-h_{1}(-x) \geqq-\|x\|-\varepsilon / 2 .
$$

Thus

$$
\sup \left\{\left|h_{1}(x)\right|: x \in E_{1}^{*}\right\} \leqq 1+\varepsilon / 2 .
$$

Proceeding by induction suppose we have $h_{n-1}, n>1$, satisfying the required properties. Define $\bar{h}_{n-1}(0)=0$ and

$$
\bar{h}_{n-1}(x)=\|x\| h_{n-1}(x /\|x\|) \text { for } x \neq 0 \text {. }
$$

By the lemma, $\bar{h}_{n-1}$ is convex, l.s.c. on $E_{1}^{*}$ and affine on $B$. Let $H_{n-1}=\left\{(x, r) \in E_{1}^{*} \times R: r \geqq \bar{h}_{n-1}+\varepsilon 2^{-n}\right\}$. If $x \in B$ and $x \neq 0$ then $\bar{h}_{n-1}(x) \geqq\|x\| f(x /\|x\|)=f(x)$. Similarly $\bar{h}_{n-1}(x) \geqq g(x)$. Since $\bar{h}_{n-1}$ is affine on $B$ it follows that $H_{n-1} \cap A=\varnothing$. Again by the separation theorem we obtain a weak* continuous affine function $h_{n}$ such that $h_{n}(x) \geqq f(x) \vee g(x)$ for $x \in B$ and $0<h_{n}(x)<\varepsilon 2^{-n}$. Also

$$
h_{n}(x) \leqq \bar{h}_{n-1}(x)+\varepsilon 2^{-n} \leqq h_{n-1}(x)+\varepsilon 2^{-n} \leqq 1+\varepsilon \sum_{k=1}^{n} 2^{-k}
$$

for all $x \in E_{1}^{*}$.

Thus $\sup \left\{\left|h_{n}(x)\right|: x \in E_{1}^{*}\right\} \leqq 1+\varepsilon \sum_{k=1}^{n} 2^{-k}$. Since $h_{n}(x) \leqq h_{n-1}(x)+\varepsilon 2^{-n}$,

$$
h_{n}(x)=2 h_{n}(0)-h_{n}(-x) \geqq-h_{n}(-x) \geqq-h_{n-1}(-x)-\varepsilon 2^{-n} .
$$

But

$$
\begin{aligned}
& -h_{n-1}(-x)=h_{n-1}(x)-2 h_{n-1}(0) \geqq h_{n-1}(x)-2 \varepsilon 2^{-(n-1)} \\
= & h_{n-1}(x)-\varepsilon 2^{-n+2} .
\end{aligned}
$$

Thus $h_{n}(x)>h_{n-1}(x)-5 \varepsilon 2^{-n}$ and so

$$
\sup \left\{\left|h_{n}(x)-h_{n-1}(x)\right|: x \in E_{1}^{*}\right\} \leqq 5 \varepsilon 2^{-n} .
$$

Define $h(x)=\lim _{n \rightarrow \infty} h_{n}(x)$. This defines an affine function $h$ on $E^{*}$ such that $h(x) \geqq f(x) \vee g(x)$ for $x \in B$ and $\sup \left\{|h(x)|: x \in E_{1}^{*}\right\} \leqq$ $1+\varepsilon=\alpha$. Since $h(0)=0$ and $h_{n} \rightarrow h$ uniformly on $E_{1}^{*}, h$ is linear and weak* continuous on $E_{1}^{*}$. Thus $h \in E$.

3. Normal cones. Let $E$ be an ordered Banach space with positive cone $P$. It will be useful in the sequel to assume that $E^{*}$ is positively generated, i.e., $E^{*}=P^{*}-P^{*}$. This property of $E^{*}$ is related to a property of $E$ called normality.

Definition. The Banach space $E$ is $\alpha$-normal if

$$
\left(E_{1}+P\right) \cap\left(E_{1}-P\right) \subset \alpha E_{1} \text {. }
$$


The space $E^{*}$ is $\alpha$-generating if $E_{1}^{*} \subset \alpha \operatorname{conv}(B \cup-B)$, where $B=$ $P^{*} \cap E_{1}^{*}$.

We now list for future reference some easy and/or well-known equivalences of 1-normality.

THEOREM 2. Let $E$ be an ordered Banach space with closed positive cone $P$. Let $B=P^{*} \cap E_{1}^{*}$. The following are equivalent.

(i) $E$ is 1-normal

(ii) $\quad x \in E$ implies $\|x\|=\sup \{|f(x)|: f \in B\}$

(iii) $E$ is isometrically isomorphic to the space $A_{0}(B)$ of continuous affine functions on $B$ vanishing at 0 , where $B$ is given the relative weak* topology

(iv) $E^{*}$ is 1-generating.

The equivalence of (i) and (iv) is a special case of a theorem of Grosberg-Krein [8]. The proof below of (i) $\Rightarrow$ (ii) is a minor modification of the proof of 23.5 of [10].

Proof. ( i ) $\Rightarrow$ (ii). Let $B^{0}$ (the polar of $\left.B\right)=\{x \in E:|f(x)| \leqq 1$ whenever $f \in B\}$. It suffices to show $B^{0} \subset E_{1}$. Let $x \in B^{0}$ and let $r$ be any number such that $0<r<1$. If $r x \notin E_{1}+P$ then there exists an $f \in E_{1}^{*}$ such that $f(r x) \leqq \inf f\left(E_{1}+P\right)$. But then $f \in B$ and $f(r x) \leqq-1$ so that $f(x)<-1$ contradicting $x \in B^{0}$. Thus $r x \in E_{1}+P$ and similarly $r x \in E_{1}-P$, so $r x \in E_{1}$ for all $r, 0<r<1$. It follows that $x \in E_{1}$.

(ii) $\Rightarrow$ (iii). It is well-known (see for example 4.5 of [13]) that the natural map of $E$ into $A_{0}(B)$ has a norm-dense image. Since (ii) implies the map is an isometry, (iii) follows.

(iii) $\Rightarrow$ (iv). Suppose $f \in E_{1}^{*} \mid \operatorname{conv}(B \cup-B)$. Then there is an $x \in E$ such that $\|x\| \geqq f(x)>\sup \{g(x): g \in \operatorname{conv}(B \cup-B)\}=\|x\|$.

(iv) $\Rightarrow$ (i ). Let $z \in E$ and $z=x+p=y-q ; x, y \in E_{1}$ and $p, q \in P$. Then $f \in E_{1}^{*}$ implies $f=\lambda f_{1}-(1-\lambda) f_{2} ; 0 \leqq \lambda \leqq 1$ and $f_{1}, f_{2} \in B$. Then

$$
f(z)=\lambda f_{1}(x+p)-(1-\lambda) f_{2}(y-q) \geqq-1
$$

and

$$
f(z)=\lambda f_{1}(y-q)-(1-\lambda) f_{2}(x+p) \leqq 1 .
$$

Thus $|f(z)| \leqq 1$ for all $f \in E_{1}^{*}$ and hence $\|z\| \leqq 1$.

Davies [4] defines an ordered Banach space to be regular if

(i) $\|y\| \leqq\|x\|$ whenever $-x \leqq y \leqq x$.

(ii) for each $\varepsilon>0$ and for each $x$ there exists $y$ such that $\|y\| \leqq\|x\|+\varepsilon$ and $y \geqq x,-x$.

He proves that if $E$ is an ordered Banach space and $E^{*}$ is given 
the dual ordering then $E^{*}$ is a Kakutani $L$-space [9] if and only if $E$ is a regular space which is directed and satisfies the Riesz decomposition property. Such spaces $E$ are termed simplex spaces by Effros [7]. We note that if $E$ is 1-normal and directed then $E$ is regular. Also, if $E^{*}$ is an $L$-space then in particular it is 1-generating and thus $E$ is 1-normal. Hence we have the following:

THEOREM 3. An ordered Banach space with closed positive cone $P$ is a simplex space if and only if

(i) E has the decomposition property,

(ii) $E$ is 1-normal, and

(iii) $E$ is directed.

4. Representation of universal caps. In the following we shall assume that $B$ is a universal cap of the convex cone $Q$ and that the space $F=Q-Q$ is a Hausdorff locally convex space considered in the weak topology $w\left(F, F^{\prime}\right)$ induced by its topological dual $F^{\prime}$. If $f \in F^{\prime}$ then define

$$
\|f\| \equiv \sup \{|f(x)|: x \in B\} .
$$

Theorem. With $\|\cdot\|$ defined as above $F^{\prime}$ is a normed linear space such that $\left(F^{\prime},\|\cdot\|\right)^{*}$ with the weak* topology is linearly homeomorphic to $F$. The dual norm induced on $F$ is additive on $Q$ and $F_{1}=\operatorname{conv}(B \cup-B)$.

This result is closely related to a theorem of Dixmier [3] which is used in the proof of the analogous result for locally compact cones in [5].

Proof. The set conv $(B \cup-B)$ is compact, convex, balanced and absorbent in $F$ and thus its Minkowsky functional is a norm on $F$ such that $F_{1}=\operatorname{conv}(B \cup-B)$. Since $Q \backslash B$ is convex, the norm is additive on $Q$. Then the polar $F_{1}^{0}$, is the unit ball for a dual norm on $F^{\prime}$ and clearly if $f \in F^{\prime}$ then $\|f\|=\sup \{|f(x)|: x \in B\}$. Since $F_{1}$ is $w\left(F, F^{\prime}\right)$ compact it follows from the Mackey-Bourbaki Duality Theorem (see 8.3.1 of [6]) that the norm topology on $F$ preserves the duality between $F^{\prime}$ and $F$, i.e., $\left(F^{\prime},\|\cdot\|\right)^{*}=F^{\prime}$. Obviously the weak* topology on $F$ is the same as $w\left(F, F^{\prime}\right)$.

CoRollary. If $B$ is a universal cap of $Q$ then $B$ absorbs any other cap $B^{\prime}$ of $Q$ (there exists $r>0$ such that $B^{\prime} \subset r B$ ).

Proof. Let $Q^{\prime}=\bigcup_{n=1}^{\infty} n B^{\prime}$. Clearly $B^{\prime}$ is a universal cap of $Q^{\prime}$ 
and $B \cap Q^{\prime}$ is also a universal cap of $Q^{\prime}$. Thus it suffices to consider the case where both $B$ and $B^{\prime}$ are universal caps of $Q$. In this case $F=Q-Q$ can be normed so that $F_{1}=\operatorname{conv}\left(B^{\prime} \cup-B^{\prime}\right)$. Since $F$ is the dual of a normed linear space $F$ is complete in this norm. Also $F=\bigcup_{n=1}^{\infty} n \operatorname{conv}(B \cup-B)$ and so it follows from the Baire theorem that $F_{1}=\operatorname{conv}\left(B^{\prime} \cup-B^{\prime}\right) \subset r \operatorname{conv}(B \cup-B)$ for some $r>0$. Suppose $x \in B^{\prime}$. Then $x=r\left(\lambda b_{1}-(1-\lambda) b_{2}\right) ; 0 \leqq \lambda \leqq 1$ and $b_{1}, b_{2} \in B$. Let $b=r \lambda b_{1}$. Then $b \in r B$ and $b-x \in Q$. If $x \neq b$ choose $\mu \geqq 1$ sufficiently large so that $x+\mu(b-x) \notin r B$. Then

$$
b=(1-1 / \mu) x+(1 / \mu)(x+\mu(b-x)) .
$$

Thus $x \in r B$ since $Q \backslash r B$ is convex. Hence $B^{\prime} \subset r B$.

It was pointed out by I. Namioka that this corollary can be proved directly from 10.2 of [10].

THEOREM. Let $B$ be a universal cap of $Q$ and let $F$ and $F^{\prime}$ be as above. Then $E=A_{0}(B)$ is the $\|\cdot\|$-completion of $F^{\prime}$. Let $E$ be ordered with closed positive cone $P$ as a subspace of $C(B)$. Then $E$ is 1-normal, approximately directed and $E^{*}$ is isometrically isomorphic to $(F,\|\cdot\|)$. This isomorphism is order preserving.

Proof. It follows from the definition of the norm on $F^{\prime}$ and a well-known theorem that $F^{\prime}$ is isometrically-isomorphic to a normdense subspace of $A_{0}(B)$. Thus $E$ is the $\|\cdot\|$-completion of $F^{\prime}$. Since $F$ is the norm dual of $F^{\prime}$ it is clear that each $x \in F$ can be uniquely extended to a bounded linear functional on $E$ with the same norm. Thus $(F,\|\cdot\|)$ is identified with $E^{*}$. To show the identification is order preserving it suffices to show $P^{*}=Q$. If $0 \neq x \in Q$ then $r x \in B$ for some $r>0$. Thus $f(x) \geqq 0$ for all $f \in P$ and so $x \in P^{*}$. Since $B$ is $w\left(F, F^{\prime}\right)$ compact it is a $w(F, E)$ closed subset of $F_{1}$ and hence $w(F, E)$ compact. But then $Q \cap F_{1}=B$ is $w(F, E)$ compact and so by the Krein-Smulian theorem $Q$ is $w(F, E)$ closed. Suppose now $x \in P^{*} \mid Q$. Then there is an $f \in E$ such that $f(x)<\inf \{f(y): y \in Q\}$. Thus $f(y) \geqq 0$ for all $y \in B$ and so $f \in P$. But inf $\{f(y): y \in Q\}=0$ and so $f(x)<0$ which contradicts $x \in P^{*}$. Thus $P^{*}=Q$. Since the norm on $F$ is additive on $Q=P^{*}, E$ is approximately directed. Also, $F_{1}=\operatorname{conv}(B \cup-B)$ and so $F$ is 1-generating and hence $E$ is 1-normal.

CoROLlaRy. If $B$ is universal cap of $Q$ then there exists an ordered Banach space $E\left(=A_{0}(B)\right)$ with closed positive cone $P$ such that $E$ is approximately directed, 1-normal and $B$ is affinely homeomorphic to $P^{*} \cap E_{1}^{*}$. If $Q$ is identified with $P^{*}$ then $Q$ is closed in the weak* topology. 
We remark here that if $Q$ has a compact base then $Q$ is closed (this is due to Klee [11]). The corresponding fact about $Q$ in the case that it has a universal cap is also true for the weak* topology as the dual of complete space but does not hold in general. We give an example below.

5. Examples. The following example shows that an approximately directed Banach space is not necessarily directed.

Let $c_{0}$ be the space of sequences converging to 0 with the supremum norm. Let

$$
E=\left\{x \in c_{0} \mid x_{1}+x_{2}=\sum_{n=0}^{\infty} 2^{-n} x_{n+3}\right\} .
$$

Clearly $E$ is a closed subspace of $c_{0}$. Let $\alpha>1$ be given and let $x, y \in E_{1}$. Choose $z_{n}=\alpha$ for $3 \leqq n \leqq N$ where $N$ is sufficiently large so that $\alpha \sum_{n=0}^{N} 2^{\cdots n} \geqq 2$. Let $z_{n}=x_{n} \vee y_{n} \vee 0$ for $n>N$. Then

$$
2 \leqq \sum_{n=0}^{\infty} 2^{-n} z_{n+3} \leqq 2 \alpha \text {. }
$$

Thus $z_{1}$ and $z_{2}$ can be chosen between 1 and $\alpha$. Then $\|z\| \leqq \alpha$ and $z_{n} \geqq x_{n} \vee y_{n}$ for all $n$. Thus $E$ is approximately directed. Now let

$$
x=(1,0,1,0, \cdots, 0, \cdots)
$$

and

$$
y=(0,1,1,0, \cdots, 0, \cdots) .
$$

If $z \geqq x, y$ and $\|z\| \leqq 1$ it would be necessary for $z_{n}$ to be exactly 1 for all $n$. But $z_{n} \rightarrow 0$ so $E$ is not 1 -directed.

We now give an example to show that a universally capped cone need not be closed. Let $P$ be the positive cone of $l^{1}$ with the product topology as a subspace of $R^{N}$. Let

$$
Q=\left\{x \in P: x_{1}=\sum_{n=2}^{\infty} x_{n} / n\right\} \text {. }
$$

Then $Q$ is not closed, for let $a^{n}$ be the element of $Q$ for which $a_{1}^{n}=1$, $a_{n}^{n}=n$ and $a_{m}^{n}=0$ for $m \neq 1, n$. Then $a^{n} \rightarrow(1,0, \cdots, 0, \cdots) \notin Q$. But since $(-1,1 / 2,1 / 3, \cdots, 1 / n, \cdots) \in c_{0}, Q$ is closed in the weak* topology on $l^{1}$ as the dual of $c_{0}$. Thus $B=\left\{x \in Q: \sum_{n=1}^{\infty} x_{n} \leqq 1\right\}$ is weak* compact and hence compact in the weaker product topology. Thus $B$ is a universal cap of $Q$.

6. Šilov boundaries. If $X$ is a compact Hausdorff space and $E$ is a subspace of the space $C(X)$ of continuous real-valued functions 
on $X$, then the subset $K$ of $X$ is called a boundary of $E$ if $f \in E$ implies there exists $x \in K$ such that $|f(x)|=\|f\|$. If there is a smallest closed boundary of $E$ (one which is contained in every other closed boundary) it is called a Silov boundary. It is well known that if $E$ separates points and contains the constants then $E$ possesses a Silov boundary. However this is not necessarily the case for arbitrary separating subspaces of $C(X)$ (consider, for example, the subspace of $C[0,1]$ of functions satisfying $f(x)+f(1-x)=0$ which possesses the two minimal closed boundaries $[0,1 / 2]$ and $[1 / 2,1])$. This is perhaps most relevant to the consideration of subspaces of $C_{0}(Y), Y$ locally compact Hausdorff; or equivalently to subspaces of continuous functions all of which vanish at some point of a compact Hausdorff space. We observe here that the usual arguments in case $E$ contains the constants can be adapted to show that a closed approximately directed subspace $E$ of $C(X)$, and consequently any dense subspace of $E$, possesses a Šilov boundary.

Let $E$ be a closed, separating and approximately directed subspace of $C(X)$; let $B=\left\{L \in E^{*}: L \geqq 0\right.$ and $\left.\|L\| \leqq 1\right\}$. Then $B$ is weak* compact and if $\phi$ is the usual evaluation map from $X$ to $E^{*}$ then $\phi(X)$ is a closed subset of $B$ homeomorphic to $X$.

\section{Proposition. $B=\operatorname{cl}-\operatorname{conv}(\phi(X) \cup\{0\})$.}

Proof. Let $K=$ cl-conv $(\phi(X) \cup\{0\})$. Obviously $K \subset B$. Suppose $L \in B \backslash K$ and without loss, $\|L\|=1$. Then $L \notin \operatorname{conv}(K \cup-K)$ : If $L \in \operatorname{conv}(K \cup-K)$ then $L=L_{1}-L_{2} ; L_{1}, L_{2} \in K$ and $\left\|L_{1}\right\|+\left\|L_{2}\right\|=1$. But since $E$ is approximately directed and

$$
L, L_{1}, L_{2} \geqq 0,1=\|L\|=\left\|L_{1}\right\|-\left\|L_{2}\right\| \text {. }
$$

Thus $\left\|L_{2}\right\|=0$. But then there exists $f \in E$ such that

$$
\|f\| \geqq L(f)>\sup \left\{L^{\prime}(f): L^{\prime} \in \operatorname{conv}(K \cup-K)\right\}=\|f\| \text {. }
$$

Definition. The Choquet boundary $C$ of

$$
E=\{x \in X: 0 \neq \phi(x) \in \operatorname{ext} B\}
$$

(ext $B=$ extreme point of $B$ ).

Since $E$ is separating there is at most one point $x_{0}$ of $X$ such that $f\left(x_{0}\right)=0$ for all $f \in E$. It can be shown with the standard arguments (see, e.g., [13]) that $C$ is in fact a boundary and that $C$ consists exactly of the points $x$ (excluding $x_{0}$ ) of $X$ for which $\varepsilon_{x}$ is the only probability measure which represents $x$ in $E$.

THEOREM. The closure of $C$ in $X$ is the Šilov boundary of $E$. 
Proof. (see also Prop. 6.4 of [13]). Let $K$ be a closed boundary of $E$ and suppose $0 \neq \phi(x) \in \operatorname{ext} B \backslash \phi(K)$. Let

$$
U=\left\{L \in B:\left|L\left(f_{i}\right)-f_{i}(x)\right|<\varepsilon ; i=1, \cdots, n\right\}
$$

be a weak* neighborhood relative to $B$ containing $\phi(x)$ and disjoint from $\phi(K)$. Let $J_{i}=\left\{L \in B:\left|L\left(f_{i}\right)-f_{i}(x)\right| \geqq \varepsilon\right\} ; i=1, \cdots, n$ and let $J=$ conv $\left(J_{1}, \cdots, J_{n},\{0\}\right)$. Then $J$ is compact, convex and contains $\phi(K)$. Since $\phi(x)$ is an extreme point of $B$ missing $J_{1}, \cdots, J_{n}$ and $\{0\}$, $\phi(x) \notin J$. Also $\|\phi(x)\|=1$ and it follows from the additivity of the norm on $B$ that $\phi(x) \notin \operatorname{conv}(J \cup-J)$. Thus there exists $f \in E$ such that

$$
f(x)>\sup \{L(f): L \in \operatorname{conv}(J \cup-J)\} \geqq \sup \{|f(y)|: y \in K\}
$$

which contradicts the fact that $K$ is a boundary.

Added in proof. Theorem 1, together with the applications in Sections 3 and 4 , has been obtained independently by Kung-Fu $\mathrm{Ng}$ [The duality of partially ordered Banach spaces, Proc. London Math. Soc. (to appear)].

\section{REFERENCES}

1. L. Asimow, Extremal structure of well-capped convex sets, Trans. Amer. Math. Soc. (to appear)

2. G. Choquet, Ensembles et cones convexes faiblement, C. R. Acad. Sci. Paris 254 (1962), 1908-1910.

3. J. Dixmier, Sur un theoreme de Banach, Duke Math. J. 15 (1948), 1057-1071.

4. E. B. Davies, On the Banach duals of certain spaces with the Riesz decomposition property, Quarterly J. Math. 18 (1967), 109-111.

5. D. A. Edwards, On the homeomorphic embedding of a locally compact cone into a Banach dual space endowed with the rague topology, Proc. London Math. Soc. (3) 14 (1964), 399-414.

6. R. E. Edwards, Functional analysis, Holt, Rinehart and Winston, New York, 1965.

7. E. G. Effros, Structure in simplexes, Acta. Math. 117 (1967), 103-121.

8. J. Grosberg and M. Krein, Sur las decomposition des functionelles en composantes positives, C. R. (Doklady) de l'Acad. Sci. de 1 URSS 25 (1939), 723-26.

9. S. Kakutani, Concrete representation of abstract $(L)$-spaces and the mean ergodic theorem, Ann. of Math. 42 (1941), 523-537.

10. Kelley, Namioka and co-authors, Linear topological spaces, van Nostrand, Princeton, 1963.

11. V. L. Klee, Jr., Separation properties of convex cones, Proc. Amer. Math. Soc. 6 (1955), 313-318.

12. M. Krein, Sur las decomposition minimale d'une functionelle linéaire en composantes positives, C. R. (Doklady) de l'Acad. Sci. de l'URSS 28 (1940), 18-24.

13. R. R. Phelps, Lectures on Choquet's theorem, van Nostrand, Princeton, 1966.

Received September 7, 1967. This research conducted while the author held a National Science Foundation Post-doctoral Fellowship. 



\section{PACIFIC JOURNAL OF MATHEMATICS}

\section{EDITORS}

\section{H. ROYDEN}

Stanford University

Stanford, California

R. R. Phelps

University of Washington

Seattle, Washington 98105

\section{J. DugundJI}

Department of Mathematics

University of Southern California

Los Angeles, California 90007

RICHARD ARENS

University of California

Los Angeles, California 90024

\section{ASSOCIATE EDITORS}
E. F. BeCKENBACH
B. H. NeumanN
F. WOLF
K. YosIDA

\section{SUPPORTING INSTITUTIONS}

\author{
UNIVERSITY OF BRITISH COLUMBIA \\ CALIFORNIA INSTITUTE OF TECHNOLOGY \\ UNIVERSITY OF CALIFORNIA \\ MONTANA STATE UNIVERSITY \\ UNIVERSITY OF NEVADA \\ NEW MEXICO STATE UNIVERSITY \\ OREGON STATE UNIVERSITY \\ UNIVERSITY OF OREGON \\ OSAKA UNIVERSITY \\ UNIVERSITY OF SOUTHERN CALIFORNIA
}

\author{
STANFORD UNIVERSITY \\ UNIVERSITY OF TOKYO \\ UNIVERSITY OF UTAH \\ WASHINGTON STATE UNIVERSITY \\ UNIVERSITY OF WASHINGTON \\ AMERICAN MATHEMATICAL SOCIETY \\ CHEVRON RESEARCH CORPORATION \\ TRW SYSTEMS \\ NAVAL WEAPONS CENTER
}

Mathematical papers intended for publication in the Pacific Journal of Mathematics should be in typed form or offset-reproduced, double spaced with large margins. Underline Greek letters in red, German in green, and script in blue. The first paragraph or two must be capable of being used separately as a synopsis of the entire paper. It should not contain references to the bibliography. Manuscripts, in duplicate if possible, may be sent to any one of the four editors. All other communications to the editors should be addressed to the managing editor, Richard Arens, University of California, Los Angeles, California 90024.

Each author of each article receives 50 reprints free of charge; additional copies may be obtained at cost in multiples of 50 .

The Pacific Journal of Mathematics is published monthly. Effective with Volume 16 the price per volume (3 numbers) is $\$ 8.00$; single issues, $\$ 3.00$. Special price for current issues to individual faculty members of supporting institutions and to individual members of the American Mathematical Society: $\$ 4.00$ per volume; single issues $\$ 1.50$. Back numbers are available.

Subscriptions, orders for back numbers, and changes of address should be sent to Pacific Journal of Mathematics, 103 Highland Boulevard, Berkeley 8, California.

Printed at Kokusai Bunken Insatsusha (International Academic Printing Co., Ltd.), 7-17, Fujimi 2-chome, Chiyoda-ku, Tokyo, Japan.

PUBLISHED BY PACIFIC JOURNAL OF MATHEMATICS, A NON-PROFIT CORPORATION

The Supporting Institutions listed above contribute to the cost of publication of this Journal, but they are not owners of publishers and have no responsibility for its content or policies. 


\section{Pacific Journal of Mathematics}

\section{Vol. 26, No. $3 \quad$ BadMonth, 1968}

Leonard Asimow, Universally well-capped cones ................. 421

Lawrence Peter Belluce, William A. Kirk and Eugene Francis Steiner,

Normal structure in Banach spaces ..................... 433

William Jay Davis, Bases in Hilbert space.................... 441

Larry Lee Dornhoff, p-automorphic p-groups and homogeneous

algebras..................................... 447

William Grady Dotson, Jr. and W. R. Mann, A generalized corollary of the

Browder-Kirk fixed point theorem ....................... 455

John Brady Garnett, On a theorem of Mergelyan ................. 461

Matthew Gould, Multiplicity type and subalgebra structure in universal

algebras............................................. 469

Marvin D. Green, A locally convex topology on a preordered space . . . . . 487

Pierre A. Grillet and Mario Petrich, Ideal extensions of semigroups . . . . . . 493

Kyong Taik Hahn, A remark on integral functions of several complex

variables ... . . . . . . . . . . . . . . . . . . . . . . . . . . . . . . . . . . . 509

Choo Whan Kim, Uniform approximation of doubly stochastic

operators..................................... 515

Charles Alan McCarthy and L. Tzafriri, Projections in $\mathscr{L}_{1}$ and

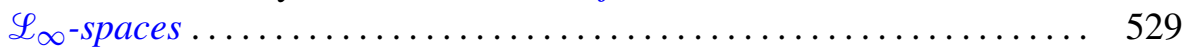

Alfred Berry Manaster, Full co-ordinals of RETs ................ 547

Donald Steven Passman, $p$-solvable doubly transitive permutation

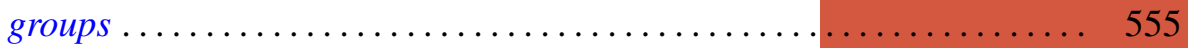

Neal Jules Rothman, An $L^{1}$ algebra for linearly quasi-ordered compact

semigroups ....................................... 579

James DeWitt Stein, Homomorphisms of semi-simple algebras .......... 589

Jacques Tits and Lucien Waelbroeck, The integration of a Lie algebra

representation ...............................

David Vere-Jones, Ergodic properties of nonnegative matrices. II ........ 601

Donald Rayl Wilken, The support of representing measures for $R(X) \ldots \ldots 621$

Abraham Zaks, Simple modules and hereditary rings .... . . 